\title{
*THE RECORD OF A LEPROUS VILLAGE
}

\author{
E. Mulr and K. R. ChatTheja.
}

Before conducting a carefully planned and controlled statistical survey of the endemiology of a disease, it is necessary to make a preliminary enquiry in a carefully chosen and limited area, so as to arrive at a rough idea of the factors influencing the spread of the disease. This preliminary enquiry should furnish the data upon which the larger statistical survey may be based.

The village of Debipur Hir in the Bankura District of Bengal was founded about 74 years ago. It consists entirely of Mussalman families, most of which are inter-related. The records cover five generations. The first two generations were free from leprosy. The disease was apparently introduced by cases 26, 34 and I20 (see tables I and II), the first two of whom were infected by their maternal uncle in a neighbouring village, and the latter by the same individual, her husband's maternal uncle. Her husband (no. 2I) probably escaped infection by being past the very susceptible age; he was 6 years older than his brother (no. 26). Case no. I20 was separated from her husband after leprosy developed, but she infected her daughter (no. 22). The latter (no. 22) however did not become infectious till her daughter (no. I5) had been married and left the village, thus escaping infection.

During the last 40 years the number of infectious cases present in the village varied as shown in the following list :-

No. of infec-

tious cases.

\begin{tabular}{llllllll} 
& \multicolumn{4}{c}{ tious cases. } & \multicolumn{2}{c}{ tious cases. } \\
I894-I898 & $\ldots$ & $\ldots$ & I & I9I2-I9I9 & $\ldots$ & $\ldots$ & I \\
I898-I899 & $\ldots$ & $\ldots$ & o & I9I9-I922 & $\ldots$ & $\ldots$ & 3 \\
I899-I905 & $\ldots$ & $\ldots$ & I & I922-I926 & $\ldots$ & $\ldots$ & 7 \\
I905-I909 & $\ldots$ & $\ldots$ & 3 & I926-I932 & $\ldots$ & $\ldots$ & 9 \\
I909-I9I2 & $\ldots$ & $\ldots$ & 2 & I932-I934 & $\ldots$ & $\ldots$ & Io \\
\hline
\end{tabular}

* The first part of a paper reprinted from Leprosy in India, VII-r, Jan. I935. 


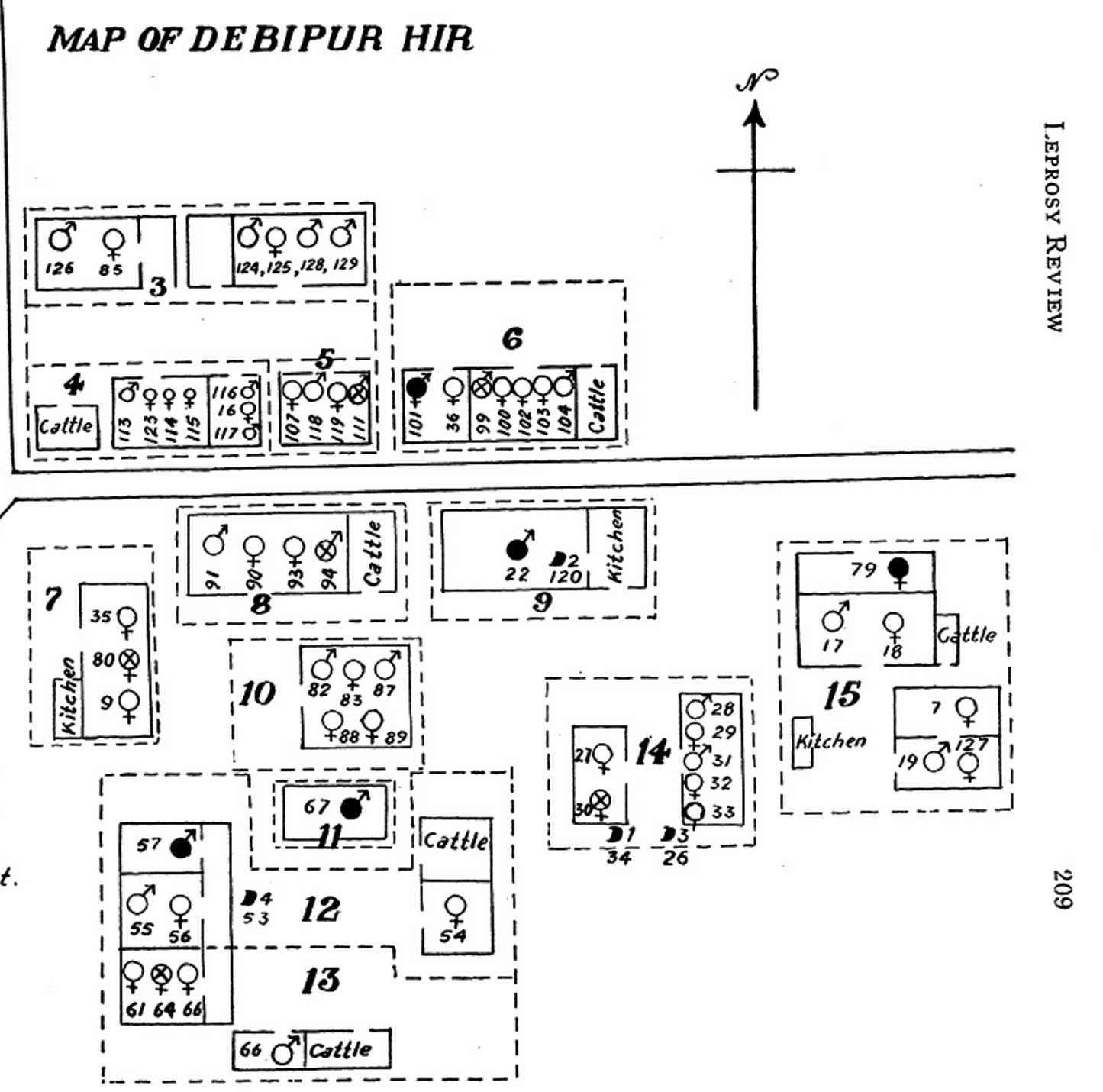


The disease began in the third generation, and within the last 46 years 23 cases have developed in the village. Of these, 4 are dead. The remaining 19 cases may be classified as follows :-

$$
\begin{aligned}
& \begin{array}{lll}
\mathrm{N}-\mathrm{I} & \ldots & 3 \\
\mathrm{~N}-2 & \ldots & 6
\end{array}
\end{aligned}
$$

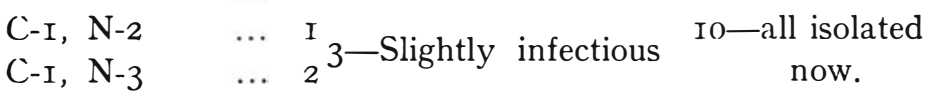

$$
\begin{aligned}
& \mathrm{C}-2 \quad \ldots \quad 4 \\
& \mathrm{C}-2, \mathrm{~N}-2 \quad \ldots \quad 27 \text {-Highly infectious } \\
& \mathrm{C}-3 \quad \ldots \quad \mathrm{I}
\end{aligned}
$$

At the present time there are 77 people in the village divided into I5 Mussalman families (see map), so that the present incidence of the disease is 24.6 per cent.

The village is a compact one and the houses are closely set together. The people are mostly day labourers, but a few cultivate their own land. The economic condition of the village is poor. There is a scarcity of drinking water and malaria is rife.

Two of the important factors in the spread of the disease are apparently family relationship and closeness of residence, both of these determining to a certain extent the degree of contact. To show the former a family tree of the two principal families has been prepared, while the latter is shown in the map of the village. The numbers in the tree and map correspond to the serial number in the general list of inhabitants (table I). This general list also gives various particulars regarding the villagers.

A second list (table II) deals with the leprous cases, and gives further details as to how and when the disease was acquired and the type of the disease at the time of examination.

Much of the historical information recorded is only approximate, as it was ascertained by cross examination of the villagers. This is particularly so with regard to the dates when cases of leprosy reached the infectious stage of the disease.

\section{Discussion.}

In looking down the source-of-infection column in table II, one is struck by the number of cases infected by case no. 67, no less than 15 in all, though in two of these (nos. 53 and $7 \mathrm{I}$ ) case 34 was possibly a previous source of infection.

Of the 13 cases who were in continuous infectious contact from birth onwards, 6 have already become infectious cases, the estimated average age of their becoming infectious being $17 \frac{1}{2}$ years. The remaining 7 cases are still non-infectious, but their average age at present is only $12 \frac{1}{2}$; so that, giving them the same period of time 
to become infectious, they have still 5 years. For the 6 infectious cases there was on an average a 6-year period between the first noticed lesions and their becoming infectious; but the 7 noninfectious cases only showed their lesions less than 5 years ago on

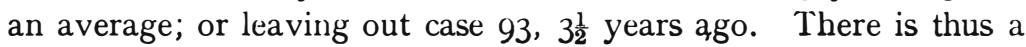
distinct tendency for those having infectious contact from birth to become infectious cases.

Cases 40, 53 and $7 \mathrm{I}$ apparently had their first infectious contacts after the age of Io, and yet they developed into infectious cases; but these 3 senior cases may quite possibly have come in contact with other sources of infection at earlier ages.

Of the non-infectious cases, no. 93 is exceptional in that he was in infectious contact from birth, but is still only an $\mathrm{N}-2$ case at the age of 20 .

There are, of course, other factors which determine the degree of infection which takes place, viz. the length, frequency and closeness of contact, and the degree of infectiousness of the source at the time of contact.

In studying the family tree one is at once struck with the frequency of infectious leprosy in the fourth generation in the families in which the infectious form of the disease was common in the third generation. In the fifth generation the children were still too young at the time of examination to show signs of the disease to any great extent.

Not only are the children of direct descent affected but also nephews and nieces who have come in contact.

The importance of early marriage both in spreading the disease and in preventing the spread is brought out by this survey. The former is shown in the case of no. I20 already remarked on. The latter is shown in nos. 63 and 92 who probably escaped infection by leaving the village at an early age to go to their husbands' homes.

In studying the map, the effect of close contact within the confined space of a small house is well shown.

There is reason to believe that the absence of sanitation, poor economic conditions, the frequency of such weakening diseases as malaria, dysentery and syphilis, and the general ignorance of the villagers have much to do with high incidence of leprosy in this village.

It should be added that a few weeks of intensive propaganda have been sufficient to secure voluntary effective isolation of the Io infectious cases still alive in the village. Whether such isolation will be effectively maintained, and the extent to which the infection will be controlled thereby in future, must be a subject for future record. But the fact that a single infectious case, such as no. 67, 
FAMILY TREE OF DEBIPUR HIR

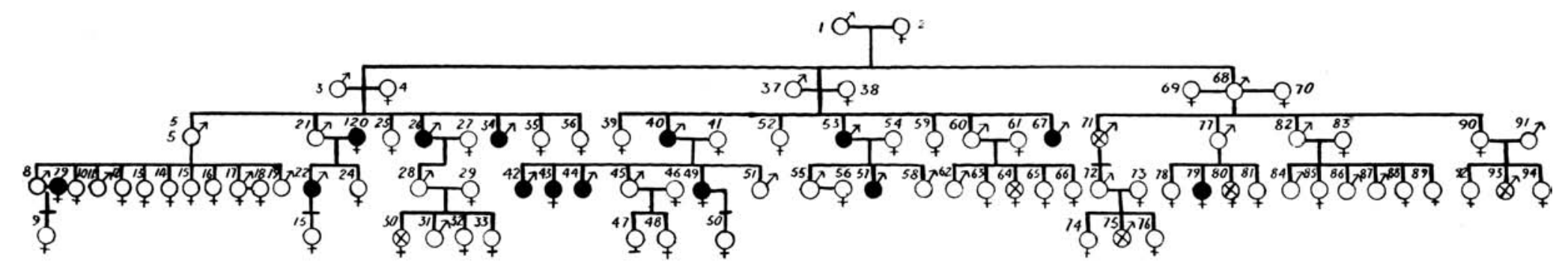

7 Infectious case of leprosy.

$\otimes$ Non-infectious - do.

O' + Non-leprous

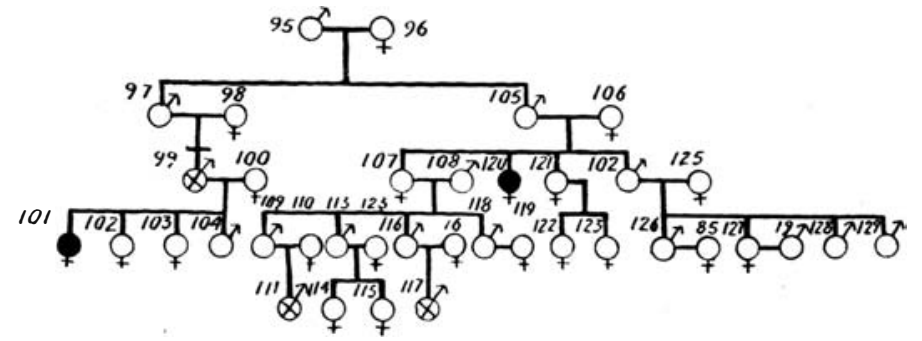


can broadcast the disease throughout the community, shows the all-importance of a careful survey followed by persistent special propaganda with a view to securing the effective isolation of all infectious cases.

Above all, considering the frequency with which those in contact from birth or in early age develop the infectious form of the disease, we would emphasise the importance of separating young children from all chance of infection.

TAIBLE I

\begin{tabular}{|c|c|c|c|c|c|c|c|}
\hline \multirow{3}{*}{$\begin{array}{l}\text { Serial } \\
\text { No. } \\
\mathrm{I}\end{array}$} & & \multirow{2}{*}{\multicolumn{2}{|c|}{$\begin{array}{l}\text { General } \\
\text { Sex }\end{array}$}} & \multicolumn{3}{|c|}{ List of Inhabitants } & \multirow{2}{*}{$\begin{aligned} & \text { of Mcbipur } \text { Jlir. } \\
& \text { Remarks }\end{aligned}$} \\
\hline & Name & & & Born & $\begin{array}{l}\text { Mar- } \\
\text { ried }\end{array}$ & l)icel & \\
\hline & Momin & $\cdots$ & M. & $\cdots$ & $\ldots$ & $?$ & $\begin{array}{l}\text { Was one of the } 2 \text { founders of } \\
\text { the village. }\end{array}$ \\
\hline 2 & $\begin{array}{l}\text { Wife of } \\
\text { Momin }\end{array}$ & $\ldots$ & F. & & & ? & \\
\hline 3 & Pachai & $\ldots$ & M. & $\cdots$ & $\cdots$ & ? & \\
\hline 4 & W. & $\ldots$ & $\mathrm{F}$ & $?$ & ... & ? & \\
\hline 5 & Sadulla & $\ldots$ & M. & 1850 & 1862 & 1916 & \\
\hline 6 & Kahiton & $\ldots$ & F. & 1855 & 1862 & 1897 & Was the ist wife. \\
\hline 7 & Khenti & $\cdots$ & F. & 1876 & 1900 & $\cdots$ & $\begin{array}{l}\text { Was the } 2 \text { nd wife. Came at } 24 \\
\text { years of age. }\end{array}$ \\
\hline 8 & Harmuj & $\ldots$ & M. & 1880 & ? & I93I & No. 79 is his widow. \\
\hline 9 & Leha & $\ldots$ & F. & 1930 & $\cdots$ & $\cdots$ & $\begin{array}{l}\text { Her mother is a leper but is } \\
\text { separate from her. }\end{array}$ \\
\hline Io & Etimon & $\ldots$ & $\mathrm{F}$. & 1883 & 1894 & & Left the village after marriage. \\
\hline I I & Rohr & $\cdots$ & M. & 1886 & $\ldots$ & 1896 & \\
\hline 12 & Letumon & $\ldots$ & F. & 1889 & 1905 & 1928 & $\begin{array}{l}\text { Was the wife of Rastom no. } 22 \\
\text { but left him when he became } \\
\text { an infectious leper, in } 1920 .\end{array}$ \\
\hline I3 & Khas & & $\mathrm{F}$. & I90I & 191.4 & 1915 & \\
\hline I4 & Asle & $\cdots$ & $\mathrm{F}$. & 1906 & 1918 & $\cdots$ & Left the village after marriage. \\
\hline I 5 & lo & $\ldots$ & $\mathrm{F}$ & 1906 & 1918 & $\ldots$ & Dit \\
\hline I6 & Musl & & $\begin{array}{l}\mathrm{F} \\
\mathrm{M}\end{array}$ & $\begin{array}{l}\text { I909 } \\
\text { I9II }\end{array}$ & $\begin{array}{l}1922 \\
1928\end{array}$ & $\cdots$ & Wife of Samed no. I I6. \\
\hline $\begin{array}{l}\text { I7 } \\
\text { I8 }\end{array}$ & $\begin{array}{l}\text { Serajaddi } \\
\text { Mahijan }\end{array}$ & $\cdots$ & F. & $\begin{array}{l}\text { I9II } \\
\text { I9I6 }\end{array}$ & $\begin{array}{l}1928 \\
1928\end{array}$ & $\begin{array}{l}\cdots \\
\cdots\end{array}$ & the village : \\
\hline I9 & & & & 2 & T931 & & Ma \\
\hline 20 & $P_{0} b$ & & $M$ & 1853 & $\ldots$ & I894 & Hearice to \\
\hline $2 \mathrm{I}$ & Khai & $\ldots$ & $\mathrm{M}$ & 1860 & 1874 & 1914 & $\begin{array}{l}\text { He abandoned his wife no. I } 20 \\
\text { when she became infectious. }\end{array}$ \\
\hline 22 & Rast & $\ldots$ & M. & 1887 & 5 & $\ldots$ & case. \\
\hline 23 & Lias & $\cdots$ & & I6 & 1927 & $\cdots$ & $\begin{array}{l}\text { Was taken away by her mother } \\
\text { no. I2 in } 1920 \text {. }\end{array}$ \\
\hline 24 & Mal & $\cdots$ & F. & I 890 & 2 & .. & Left village after marriage. \\
\hline $\begin{array}{l}25 \\
26\end{array}$ & & $\cdots$ & $\mathrm{F}$ & 1864 & & & Ditto \\
\hline 27 & $\begin{array}{l}\text { Earuddin } \\
\text { Hablesh }\end{array}$ & $\cdots$ & $\mathrm{F}$. & $\begin{array}{l}1866 \\
1870\end{array}$ & $\begin{array}{l}1886 \\
1886\end{array}$ & $\begin{array}{l}\mathrm{I} 9 \mathrm{I} 2 \\
\ldots\end{array}$ & $\begin{array}{l}\text { Leprosy case. } \\
\text { Came to village after marriage. }\end{array}$ \\
\hline & & & & & & & \\
\hline 28 & $\mathrm{~S}$ & $\ldots$ & M. & 899 & I9 & $\ldots$ & parate from fathe \\
\hline 29 & $\Gamma$ & $\cdots$ & 氺 & 903 & I919 & $\cdots$ & to village after $m$ \\
\hline 30 & Fati & $\cdots$ & $\mathrm{F}$ & 1926 & $\cdots$ & $\cdots$ & $\begin{array}{l}\text { Leprosy case. Pet of Rastom } \\
\text { (22). }\end{array}$ \\
\hline $3 \mathrm{I}$ & & & $\mathrm{M}$ & & & & \\
\hline 32 & G & $\cdots$ & $\mathrm{F}$ & & $\cdots$ & $\cdots$ & \\
\hline $\begin{array}{l}33 \\
34\end{array}$ & $\begin{array}{l}\text { Aleshi } \\
\text { Jiaruddi* }\end{array}$ & $\cdots$ & M. & 1868 & $\cdots$ & 1088 & First leper in village \\
\hline 35 & Ruta & .. & $\mathrm{F}$ & 187 & $\cdots$ & $\ldots$ & Wife of Abid no. 7 \\
\hline 36 & Uta & & & I874 & & 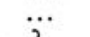 & Wife of Misir no. 99 \\
\hline 37 & Abbas & $\cdots$ & $\mathrm{M}$ & $\cdots$ & $\cdots$ & ? & \\
\hline 38 & Ugan & $\cdots$ & $\mathbf{F}$ & $\cdots$ & $\cdots$ & I9I4 & \\
\hline
\end{tabular}




\begin{tabular}{|c|c|c|c|c|c|c|c|}
\hline Serial & Name & & Sex & Born & Mar- & Jied & Remarks \\
\hline 39 & Rohiton & & & $185^{8}$ & $\begin{array}{l}\text { rieci } \\
1872\end{array}$ & ? & Left village after marriage. \\
\hline 40 & Madhu* & $\cdots$ & M. & 1864 & 1889 & $\ldots$ & Leprosy case. \\
\hline $4 \mathrm{I}$ & Koiton & ... & F. & I872 & 1889 & ... & Came to village after marriage. \\
\hline 42 & Surmail* & $\cdots$ & M. & I894 & 191.4 & ... & $\begin{array}{l}\text { Leprosy case. Wife left him } \\
\text { when in infectious stage. }\end{array}$ \\
\hline 43 & Mali* & $\cdots$ & F. & I899 & $\cdots$ & ‥ & Leprosy case. \\
\hline 44 & Ismail* & ... & M. & I9OI & 1921 & $\cdots$ & $\begin{array}{l}\text { Leprosy case. His wife (I22) } \\
\text { left him. }\end{array}$ \\
\hline 45 & Osman & $\cdots$ & M. & r904 & 1922 & ... & $\begin{array}{l}\text { After birth he was reared at his } \\
\text { maternal uncle's village till } \\
\text { his marriage. Has returned } \\
\text { and living in a separate house }\end{array}$ \\
\hline 46 & Kasemon & $\cdots$ & F. & I908 & 1922 & $\cdots$ & Came to village after marriage. \\
\hline 47 & Nurjan & $\cdots$ & I. & I927 & ... & ... & Lives separately from lepers. \\
\hline 48 & Moharjan & $\ldots$ & F. & I933 & $\cdots$ & $\cdots$ & Ditto. \\
\hline 49 & Rupi* & $\cdots$ & I. & 1907 & 1919 & $\cdots$ & $\begin{array}{l}\text { Leprosy case. Was married but } \\
\text { husband divorced her at her } \\
\text { infectious stage. }\end{array}$ \\
\hline 50 & Nekjan & $\cdots$ & $\mathrm{F}$. & I929 & ... & $\cdots$ & $\begin{array}{l}\text { Living separate from mother } \\
(49) \text {. }\end{array}$ \\
\hline $5 \mathrm{I}$ & Samsuddi & ... & M. & I9I 2 & ... & $\cdots$ & $\begin{array}{l}\text { Born and brought up atmaternal } \\
\text { uncle's village and still there. }\end{array}$ \\
\hline 52 & Nuniton & $\cdots$ & $\mathrm{F}$. & 1870 & 1884 & 1926 & Left village after marriage. \\
\hline 53 & Ali* & ... & M. & I880 & 1898 & I926 & Leprosy case. \\
\hline 54 & Khurison & ... & F. & I 886 & 1898 & $\cdots$ & $\begin{array}{l}\text { Came to the village after marri- } \\
\text { age. Had no intimate contact } \\
\text { with any infectious leper. }\end{array}$ \\
\hline 55 & Asijaddi & $\cdots$ & M. & I905 & 1925 & $\cdots$ & \\
\hline 56 & Kar & $\cdots$ & $\mathrm{F}$. & I9IO & I925 & $\cdots$ & Came to village after marriage. \\
\hline 57 & Taimur* & $\cdots$ & M. & I9IO & $\cdots$ & $\cdots$ & Leprosy case. \\
\hline 58 & Hamed & ... & M. & I9I2 & $\cdots$ & $\cdots$ & $\begin{array}{l}\text { Was at another village from his } \\
\text { infancy. }\end{array}$ \\
\hline $\begin{array}{l}59 \\
60\end{array}$ & $\begin{array}{l}\text { Aharan } \\
\text { Sadaraddi }\end{array}$ & $\cdots$ & $\mathrm{F}$. & 1882 & 189.4 & .. & Wife of Ataulla no. $7 \mathrm{I}$. \\
\hline $6 I$ & Khusmon & ... & $\mathrm{F}$. & $\begin{array}{l}1004 \\
1893\end{array}$ & 1908 & $\cdots$ & Came to village after marriage. \\
\hline 62 & Hatem & ... & M. & I9I2 & ... & 1919 & \\
\hline 63 & Sab & $\ldots$ & F. & I9I6 & 1928 & $\ldots$ & Left the village after marriage. \\
\hline 64 & Nebura $\uparrow$ & ... & F. & I920 & ‥ & $\cdots$ & Leprosy case. \\
\hline 65 & Abjura & $\cdots$ & F. & I924 & $\cdots$ & 1927 & \\
\hline 66 & Guljura & ... & F. & r928 & ... & ... & $\begin{array}{l}\text { Lives separately from uncle } \\
\text { no. } 67 .\end{array}$ \\
\hline 67 & Badaraddi & & M. & I89I & $\cdots$ & & Leprosy case. \\
\hline 68 & Saifu & $\cdots$ & M. & $\cdots$ & $\cdots$ & $?$ & \\
\hline 69 & Tarifan & $\cdots$ & F. & $\cdots$ & ... & $?$ & Ist wife of 68 . \\
\hline $\begin{array}{l}70 \\
71\end{array}$ & Sairfan & ... & F. & $\ddot{8}_{5}$ & $\begin{array}{l}\cdots \\
\cdots\end{array}$ & & 2nd wife of 68 . \\
\hline $\begin{array}{l}71 \\
72\end{array}$ & $\begin{array}{l}\text { Atau } \\
\text { Abul }\end{array}$ & $\begin{array}{l}\cdots \\
\cdots\end{array}$ & M. & $\begin{array}{l}1005 \\
1904\end{array}$ & 19020 & $\cdots$ & Leprosy case. Wil \\
\hline 73 & Aklimon & $\ldots$ & $\mathrm{F}$. & I909 & , & ... & Came to village after marriage. \\
\hline 74 & Aye & . & $\mathrm{F}$. & I923 & $\cdots$ & $\cdots$ & \\
\hline 75 & med $\dagger$ & & M. & 1926 & $\cdots$ & $\cdots$ & Leprosy case. \\
\hline $\begin{array}{l}76 \\
77\end{array}$ & Ramesha & $\ldots$ & F. & I93I & $\cdots$ & $\ldots$ & \\
\hline $\begin{array}{l}77 \\
78\end{array}$ & $\begin{array}{l}\text { Abid } \\
\text { Abson }\end{array}$ & $\cdots$ & $\mathrm{M}$. & $\begin{array}{l}\text { I870 } \\
\text { I909 }\end{array}$ & $\cdots$ & $\begin{array}{l}1934 \\
\text { I922 }\end{array}$ & Wife is no. 35 \\
\hline 79 & Hati* & $\cdots$ & $\mathrm{F}$. & $\begin{array}{l}1909 \\
\text { I912 }\end{array}$ & 1924 & $\begin{array}{l}1922 \\
\ldots\end{array}$ & $\begin{array}{l}\text { Leprosy case. Widow of no. } 8 . \\
\text { Has a daughter, age } 4 \text { years, } \\
\text { separated from mother. }\end{array}$ \\
\hline 80 & Jati† & $\cdots$ & F. & I9I8 & $\ldots$ & $\cdots$ & Leprosy case. \\
\hline $8 \mathrm{I}$ & Mati & $\ldots$ & $\mathrm{F}$. & I9I 8 & I929 & $\ldots$ & Left village after marriage. \\
\hline 82 & Nazimuddi & & M. & I880 & I 898 & ... & \\
\hline 83 & Afruja & ... & F. & I886 & 1898 & ... & Came to village after marriage. \\
\hline 84 & Khalil & $\ldots$ & M. & I9I7 & ... & ... & $\begin{array}{l}\text { Born and brought up at mater- } \\
\text { nal uncle's house and still } \\
\text { there. }\end{array}$ \\
\hline 85 & Marufa & $\ldots$ & F. & I920 & I934 & ... & $\begin{array}{l}\text { Do. and came to village after } \\
\text { marriage. Husband no. I26. }\end{array}$ \\
\hline 86 & Jal & & & I923 & . & & 6 \\
\hline
\end{tabular}




\begin{tabular}{|c|c|c|c|c|c|c|c|}
\hline $\begin{array}{l}\text { Serial } \\
\text { No. }\end{array}$ & Name & & Sex & Born & $\begin{array}{l}\text { Mar- } \\
\text { ried }\end{array}$ & Died & Remarks \\
\hline 87 & Khalek & $\cdots$ & M. & 1926 & $\ldots$ & ... & \\
\hline 88 & Kudbani & $\ldots$ & F. & I929 & ... & ... & \\
\hline 89 & Puti & $\cdots$ & $\mathrm{F}$. & 1932 & ... & ... & \\
\hline 90 & Kadu & $\ldots$ & $\mathrm{F}$. & I896 & 1908 & $\cdots$ & \\
\hline 9I & Anus. & $\ldots$ & M. & I884 & 1908 & ... & Came to village after marriage. \\
\hline 92 & Kamini & $\ldots$ & $\mathrm{F}$. & I909 & I924 & ... & Ieft village after marriage. \\
\hline 93 & Elijaddi* & ... & M. & 1914 & ... & $\ldots$ & Ieeprosy case. \\
\hline 94 & Delera & $\cdots$ & $\mathrm{F}$. & I920 & ... & $\ldots$ & \\
\hline 95 & Amin & $\ldots$ & M. & ... & $\cdots$ & ? & $\begin{array}{l}\text { Was one of } 2 \text { founders of the } \\
\text { village. }\end{array}$ \\
\hline 96 & W. of 95 & $\ldots$ & $\mathrm{F}$. & $\cdots$ & $\cdots$ & ? & \\
\hline 97 & Dhiron & ... & M. & ... & $\ldots$ & ? & \\
\hline 98 & Moyna & $\ldots$ & $\mathrm{F}$ & $\cdots \cdots$ & ... & ? & \\
\hline 99 & Misir $†$ & $\cdots$ & M. & I879 & ... & $\cdots$ & Ieprosy case. Ist wife is no. 36 . \\
\hline IOO & Khusmon & $\ldots$ & F. & I908 & 192.4 & $\cdots$ & $\begin{array}{l}\text { 2nd wife of no. 99. Canie to } \\
\text { village after marriage. }\end{array}$ \\
\hline IOI & Nathi* & $\cdots$ & F. & I9I 4 & $\cdots$ & $\cdots$ & I,eprosy case. \\
\hline 102 & Ashman & $\cdots$ & F. & 1926 & $\cdots$ & $\cdots$ & \\
\hline I03 & Ajeda & $\cdots$ & $\mathrm{F}$. & 1929 & $\cdots$ & $\cdots$ & \\
\hline I04 & Sobhan & $\cdots$ & M. & I932 & $\cdots$ & $\dddot{j}$ & \\
\hline 105 & Jigru & $\cdots$ & M. & $\cdots$ & $\cdots$ & $?$ & \\
\hline 106 & Lutfan & $\cdots$ & F. & $\ddot{8}$ & $\cdots$ & ? & \\
\hline $\begin{array}{l}107 \\
\text { I08 }\end{array}$ & Duli & $\cdots$ & F. & I862 & $\cdots$ & ‥ & \\
\hline $\begin{array}{l}\text { I08 } \\
\text { ro9 }\end{array}$ & Damu & $\cdots$ & $M$. & $\cdots$ & $\cdots$ & 1928 & Came to village after marriage. \\
\hline $\begin{array}{l}\text { I09 } \\
\text { I IO }\end{array}$ & Khusmon & $\cdots$ & $\mathrm{I}$ & $\begin{array}{l}1900 \\
1906\end{array}$ & $\begin{array}{l}1918 \\
1918\end{array}$ & 1920 & Came to village after marriage \\
\hline & & & & & & & $\begin{array}{l}\text { and left it with younger son } \\
\text { after death of husband. }\end{array}$ \\
\hline I I I & Nursad $\dagger$ & $\ldots$ & M. & 1920 & $\cdots$ & $\cdots$ & Leprosy case. \\
\hline I I 2 & Irsad & $\ldots$ & M. & I925 & ... & $\cdots$ & Went with the mother no. r ro. \\
\hline I I 3 & Sabecl & $\ldots$ & M. & I904 & I9) 23 & $\cdots$ & Wife is no. I 23. \\
\hline I I 4 & Sakemon & $\cdots$ & $\mathrm{F}$. & I926 & $\cdots$ & $\cdots$ & \\
\hline I I 5 & Nahura & $\cdots$ & $\mathrm{F}$ & I929 & $\cdots$ & $\cdots$ & \\
\hline I I6 & Samed & $\cdots$ & M. & I908 & $19) 22$ & $\cdots$ & Wife is no. I6. \\
\hline I I 7 & Mursad $\dagger$ & $\cdots$ & M. & 1927 & $\cdots$ & $\cdots$ & I.eprosy case. \\
\hline I I 8 & Jabed & $\cdots$ & M. & I9I2 & 19.30 & $\cdots$ & \\
\hline $\begin{array}{l}\text { I I9 } \\
\text { I } 20\end{array}$ & Aslemon & $\cdots$ & $\mathrm{F}$. & I9I6 & 1930 & $\cdots$ & Came to village after marriage. \\
\hline $\begin{array}{l}\text { I } 20 \\
\text { I } 2 \text { I }\end{array}$ & Durpadi* & $\cdots$ & F. & 1872 & $\dddot{0}$ & I904 & Leprosy case. \\
\hline $\begin{array}{l}121 \\
122\end{array}$ & Bidu & $\cdots$ & F. & 1882 & 1894 & $\cdots$ & Leit the vilage arter marriage. \\
\hline & 11001 & $\cdots$ & $\boldsymbol{F}$. & 1905 & 1021 & $\cdots$ & $\begin{array}{l}\text { age and was married to no. } \\
44 \text { but left him at his } \\
\text { infectious stage. }\end{array}$ \\
\hline I23 & Udi & $\cdots$ & $\mathrm{F}$. & I9IO & 1923 & $\cdots$ & Wife of no. II3. \\
\hline 124 & Hauji & & M. & I892 & 1915 & $\cdots$ & \\
\hline 125 & Nishada & $\cdots$ & $\mathrm{F}$. & I902 & $10) 15$ & $\cdots$ & Came to village after marriage. \\
\hline I26 & Johaque & & M. & I9I6 & $\cdots$ & .. & Wife is no. 85 \\
\hline 127 & Koimon & $\cdots$ & F. & I920 & I9.30 & $\cdots$ & Wife of no. I9. \\
\hline I 28 & Mialal & $\cdots$ & M. & I922 & $\cdots$ & $\cdots$ & \\
\hline 129 & Sadiman & $\cdots$ & M. & I927 & $\cdots$ & $\cdots$ & \\
\hline
\end{tabular}

[This same village of Debipur $\mathrm{Hir}$ has been re-surveyed five years later, and we abstract from Leprosy in India, July, I939, an article by Dr. Dharmendra in which the results found are described. While it is still too soon to determine the effects of the survey, the educational campaign and the resulting partial segregation which resulted, the results are, at least, promising. -Editor]. 
TABle II

List of Lepers in Debipur Hir.

Probable

Source of age first Age and Age and

case contact ist lesion came

\begin{tabular}{|c|c|c|}
\hline Serial & & \\
\hline 22 & Rastom* & $\cdots$ \\
\hline 26 & Earuddi* & $\cdots$ \\
\hline 30 & Fati & $\ldots$ \\
\hline 34 & Jiaruddi* & $\ldots$ \\
\hline 40 & Madhu* & $\cdots$ \\
\hline $4^{2}$ & Surmail* & $\cdots$ \\
\hline 43 & Mali* & ... \\
\hline 44 & Ismail* & $\cdots$ \\
\hline 49 & Rupi* & $\cdots$ \\
\hline 53 & Ali* & $\cdots$ \\
\hline 57 & Taimur* & $\cdots$ \\
\hline 64 & Nebura & $\cdots$ \\
\hline 67 & Badaraddi* & $\cdots$ \\
\hline 71 & Ataulla* & $\cdots$ \\
\hline 75 & Mahamed $\dagger$ & $\cdots$ \\
\hline 79 & Hati* & $\cdots$ \\
\hline 80 & Jati ${ }_{\text {Fliiaddit }}$ & $\cdots$ \\
\hline $\begin{array}{l}93 \\
99\end{array}$ & $\begin{array}{l}\text { Elijaddi } \dagger \\
\text { Misirt }\end{array}$ & $\cdots$ \\
\hline & & \\
\hline IOI & Nathi* & $\cdots$ \\
\hline I I I & Nursad $\dagger$ & $\cdots$ \\
\hline I 17 & $\begin{array}{l}\text { Mursad } t \\
\text { Durpadi* }\end{array}$ & $\cdots$ \\
\hline 120 & & $\cdot$ \\
\hline
\end{tabular}

$\begin{array}{ll} & \text { Sex } \\ \ldots & \\ \ldots & \\ \ldots & \\ \ldots & \mathrm{F} \\ \cdots & \mathrm{M} \\ \ldots & \mathrm{M} \\ \ldots & \mathrm{F} \\ \cdots & \mathrm{M} \\ \ldots & \mathrm{F} \\ \cdots & \mathrm{M} \\ \ldots & \mathrm{M} \\ \cdots & \mathrm{F} \\ \cdots & \mathrm{M} \\ \cdots & \mathrm{M} \\ \ldots & \mathrm{M} \\ \cdots & \mathrm{F} \\ \cdots & \mathrm{F} \\ \cdots & \mathrm{M} \\ \cdots & \mathrm{M} \\ \ldots & \mathrm{F} \\ \cdots & \mathrm{M} \\ \cdots & \mathrm{M} \\ \cdots & \mathrm{F} \\ & \end{array}$

st lesion came

Died

Present
type

number year

noticed infectious

34
I 20

7
I 2

27-I9I4 33-I920

C-3 Syphilis.

Uncle

?
32-I $898 \quad 4 \mathrm{I}-\mathrm{I} 907$

I9I2

... Syphilis.

Uncle

birth

$6-1932$
$20-1888$

26- - 899

I $\ddot{89} 8$

$\mathrm{N}-2$

50-I9I4 58-I922

C-I, N-3 Dysentery

34
34

30

20-I9I4 25-I9I9

$\ldots$

C-2, N-2 Syphilis.

I 20

5

I I-I I IO 8 - 8 - 927

$\ldots$

$\mathrm{C}-2, \mathrm{~N}-2$ Malaria.

birth

I3-I9I4

22- 1923

...

$\mathrm{C}-2$

birth

8 - 1920

I 2-I924

I926

$\mathrm{C}-2$

I 4
25

$42-1922$

44 - 1924

.

Syph. and Gonhr.

birth II-I92I I6-I926

birth

$9-1929$

$7-\mathrm{I} 898$

$\mathrm{C}-\mathrm{I}, \mathrm{N}-2$

50-I9I5

I4-I905

... N-2

C-I, N-3

40

5-I93I

...

... N-2

birth

,"

8- I920

I 2 - I92

IO-I 928

$8-1922$

-I924

",

$49-1928$

...

.. $\quad \mathrm{N}-\mathrm{I}$

$\mathrm{N}-\mathrm{I}$
$\mathrm{C}-2$

I2O
67 birth

IO--I924

$\cdots$

.. $\mathrm{N}-2$

34

$\begin{array}{ll}\text { F. } & 67 \\ \text { M. } & 67\end{array}$

67

Uncle of
husband

$\begin{array}{lr}\text {," } & \text { IO-I930 } \\ \text { ", } & 6-1933\end{array}$

I 8-I932

... N-2

22- 894

$$
\text { .... }
$$

$\cdots$

27 - 899

I...

C-2

N-I

Infectious case

$\dagger$ Non-infectious-case.

$\frac{1}{\sigma}$ 


\section{The Re-Survey.}

The population, change in.-The population recorded at the original survey was seventy-seven. Three persons at that time living in the village were missed. Of these one is still in the village while the other two have gone out in search of employment. During the period between the original survey and the present survey there have been fifteen additions-II births, 2 migrated into the village, 2 girls came to the village after marriage. On the other hand, there has been a decrease of twelve- 5 deaths, 3 girls left the village after marriage, 4 persons gone out in search of employment. Thus the net population of the village was eighty-one on Ist April, I939.

Number of lepers, change in.-In I934 there were recorded nineteen lepers. During the period from I934 to I939 there has been one addition - a case of leprosy coming to the village after marriage. On the other hand, there have been a decrease of four -2 deaths, one left the village after marriage and one gone out for employment elsewhere.

Fresh cases.-During this period no fresh case has arisen amongst the population examined in I934. A case has been detected in one of the new comers to the village. Asleman Bibi came to the village after marriage from a neighbouring village (Badulara) which is known to have a high incidence of leprosy. Asleman's aunt was a lepromatous case who died about twelve years ago. Asleman's brother is a neural case with drop-foot and claw-hands. There are also two lepromatous cases in the neighbourhood-one on each side of her father's house at Badulara. Asleman is a lepromatous case.

Incidence of leprosy.-In I934 there were I9 cases in a population of 77 giving an incidence of $24.6 \%$. In I939 there are I6 cases in a population of $8 \mathrm{I}$ giving an incidence of $19.7 \%$.

Type distribution. In I934 the nineteen cases were classified as follows:-

\begin{tabular}{|c|c|c|c|}
\hline $\begin{array}{l}\mathrm{N}_{1} \\
\mathrm{~N}_{2}\end{array}$ & $\begin{array}{l}\ldots \\
\ldots\end{array}$ & $\begin{array}{l}3 \\
6\end{array}$ & Non-infectious. \\
\hline $\mathrm{CIN}_{2}$ & $\ldots$ & I & \\
\hline $\mathrm{CIN}_{3}$ & $\ldots$ & 2 & Slightly infectious. \\
\hline $\mathrm{C}_{2}$ & $\ldots$ & 4 & \\
\hline $\mathrm{C}_{2} \mathrm{~N}_{2}$. & $\ldots$ & 2 & Highly infectious. \\
\hline $\mathrm{C}_{3}$ & $\ldots$ & I & \\
\hline
\end{tabular}

Of the nine non-infectious cases one has since died, one has left the village after marriage, one is away on some employment and one has become infectious (a L2 case). The remaining five are still non-infectious. 
Of the three cases recorded as slightly infectious one has since died and two show no signs of any lepromatous change. Slit smears from ears, forehead and back in these two cases were negative for leprosy bacilli, smears were taken on a second occasion but they were still negative. All the seven cases recorded as highly infectious are still in that condition. A fresh lepromatous case (Asleman Bibi) has come to the village. The sixteen cases found in the village at the re-survey can be classified as under:

$\begin{array}{llllll}\mathrm{Nm} & \ldots & \ldots & \ldots & 5 & \\ \mathrm{Na} 2 & \ldots & \ldots & \ldots & \text { I } & 7 \text { non-infectious. } \\ \mathrm{Na} 3 & \ldots & \ldots & \ldots & \text { I } & \\ \mathrm{LI} & \ldots & \ldots & \ldots & \text { I } & \\ \mathrm{L} 2 & \ldots & \ldots & \ldots & 3 & \\ \mathrm{~L} 2 \mathrm{Na} 2 & & \ldots & \ldots & 4 & 9 \text { infectious. } \\ \mathrm{L} 3 & \ldots & \ldots & \ldots & \text { I } & \end{array}$

The accompanying table gives a comparative statement of the condition of the lepers in I934 and in I939. Only one of the cases (case No. 99) recorded as non-infectious in I934 has become infectious. All the other neural cases reported improvement some of them quite a marked one. In all the neuromacular cases the macules were flat and showed no sign of activity. In some cases it was definitely stated that the patches had faded a good deal and in one case (II7) there was no definite anresthesia in the patch. The lepromatous cases also reported improvement in their condition since they were last examined.

In the original survey report it was recorded that of the thirteen cases who were in continuous contact from birth onwards six had already become infectious cases, the remaining seven being still non-infectious. At the re-survey after a period of five years those seven were still found to be non-infectious. One of the six cases reported to be infectious in 1934 was found to be noninfectious at the re-survey. So that of the thirteen cases who had infectious contact since birth only five have so far become infectious. One of the neural cases (case No. 99) has in the meantime become infectious, this case probably had his first infectious contact at the age of $\mathrm{I} 6$. This brings the number of cases who had their first infectious contact after the age of ro and yet developed into infectious cases to four.

Isolation.-In the report of the original survey it is noted that through propaganda it has been possible to secure voluntary and effective isolation of the infectious cases in the village. One of the purposes of the present survey was to find out whether such isolation has been effectively maintained and if so what has been its effect on the spread of the disease in the village.

The isolation consists in the infectious patients not sharing the 
same room with the healthy persons in the family. It was also stated that their food was served in their own rooms. There is no isolation beyond this as the infectious patients were seen moving about freely in the compound of the houses and round about the village. In the centre of the village there is a shady tree. This provides a common meeting place for the villagers including the lepers and the children. The isolation thus appears to be only partial. It is too early to say to what extent will the spread of infection be controlled by this partial isolation but the findings at the re-survey appear to be rather encouraging.

In 1934 there were twenty-three persons of susceptıble ages that is of ages below I5 (5 under 3 , I 3 from 4 to 10, and 5 from II to I5) who were free from any signs of leprosy at that time. In I939 only twenty of these cases could be examined as two girls had left the village after marriage and one boy was away at his maternal uncle's village. None of the twenty persons examined showed any signs of leprosy and the villagers said that the three persons who had left the village had no sign. Eleven children were born in the village after the survey, none of them showed any sign of the disease but that does not mean anything as it is too early to expect anything in them. Two girls have come to the village after marriage, one of these is a case of leprosy. The present age of this girl is about 20 years, she came to the village after the I934 survey, i.e., after she was I5 years old. She has come from a neighbouring village Badulara by name which has a high incidence of leprosy. In this case there is a definite history of contact, her aunt was a lepromatous case, her brother is a neural case and there were two lepromatous cases in the neighbourhood. It is very probable that this girl was infected in her father's house before she came to Debipur Hir. Thus if this case be excluded there has not been a single fresh case during these five years although persons of suscêptible age were present in the village.

REFERENCE

Muir, E., and Chatterji, K. R. 1935 ... Leprosy in India, Vol. VIII, No. 4.

Note.-We are using the symbol L (lepromatous) in place of symbol C (cutaneous) according to the Cairo Leprosy Congress classification.

There are three cases which were classified as N2 in 1934 but are being classified as NI now. According to the Cairo classification a case is not necessarily to be classified as $\mathrm{N}_{2}$ simply on account of presence of lesions on more than one part of the body. All these three cases had two macules each, the macules being on the different parts of the body. The extent of the lesions, however, does not justify these cases being classified as $\mathrm{N} 2$. 
Table showing the condition of palients in 1934 and 1939.

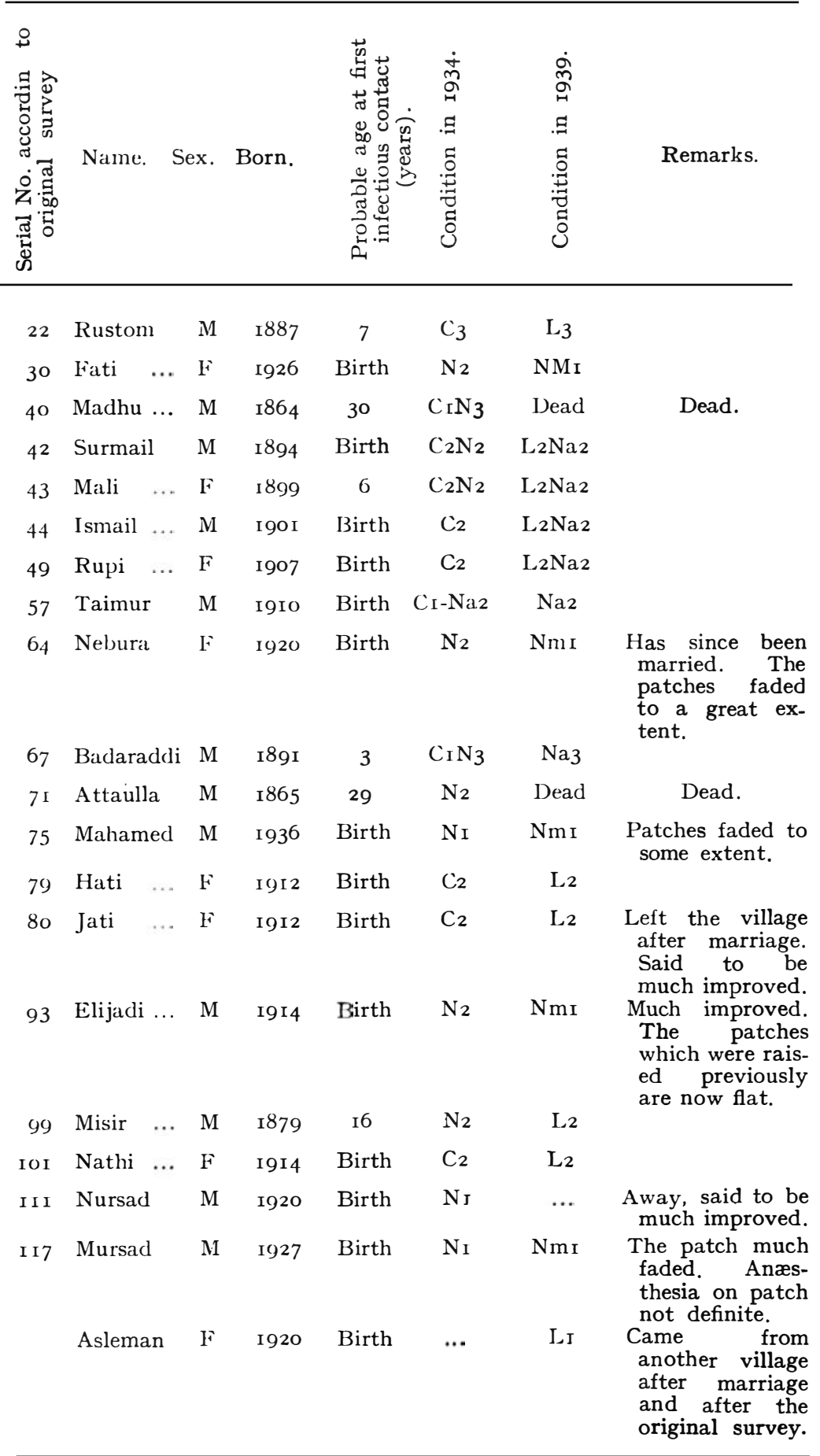

